\title{
Potassium and risk of Type 2 diabetes
}

\author{
Ranee Chatterjee $^{1, \dagger}$, Hsin-Chieh Yeh ${ }^{2}$, David Edelman ${ }^{1}$, and Frederick Brancati ${ }^{2}$ \\ ${ }^{1}$ Department of Medicine, Duke University, Durham, NC, USA \\ ${ }^{2}$ Department of Medicine and Epidemiology, Johns Hopkins University, Baltimore, MD, USA
}

\begin{abstract}
The rising incidence and prevalence of Type 2 diabetes worldwide requires us to try to identify the determinants of this epidemic and to identify improved measures to prevent and treat this condition. While obesity is a major risk factor for diabetes, there are other risk factors that could potentially be corrected more easily. Potassium, both serum levels and to a lesser extent dietary intake levels, has been associated with incident diabetes. Lower levels of potassium have been found to be associated with a higher risk of diabetes in some studies. This article will review the literature available describing these associations and will help to identify where further research is needed.
\end{abstract}

\section{Keywords}

antihypertensives affecting potassium; diabetes risk; dietary potassium; serum potassium

Type 2 diabetes mellitus imposes a growing public health burden worldwide, leading to complications, disability, mortality and higher healthcare costs $[1,2]$. While obesity is probably the most important explanatory factor $[3,101]$ there are other metabolic, environmental and genetic factors that contribute to the diabetes epidemic [4-6]. Potassium deficiency is a well-established correlate of disturbances in glucose metabolism. However, this association has been best studied in uncommon conditions, such as hyperaldosteronism [7,8], or in the context of medication use, particularly thiazide diuretics [9-11]. Indeed, it is worth noting that the rise in the incidence of diabetes is concomitant with a rise in the use of thiazide diuretics [12]. Recent research has led to renewed interest in low potassium as a possible risk factor for diabetes that is both common and potentially modifiable $[13,14]$. This article will briefly review the literature regarding potassium and diabetes risk in adults.

\section{Potassium in humans}

Potassium is the main intracellular cation in the human body and is required for vital cellular processes. Serum and dietary potassium are measurements that can affect total body stores of potassium, which can only be accurately measured using whole body counters with radioactively labeled potassium. Serum potassium is tightly controlled through homeostatic mechanisms and is affected by many factors including dietary potassium intake, potassium excretion (which is primarily in the urine) and by factors that affect potassium excretion and

\footnotetext{
(C) 2011 Expert Reviews Ltd

†Author for correspondence: Tel.: +1 919361 6103, Fax: +1 9195440934 ranee.chatterjee@ duke.edu.

Financial \& competing interests disclosure: The authors have no other relevant affiliations or financial involvement with any organization or entity with a financial interest in or financial conflict with the subject matter or materials discussed in the manuscript apart from those disclosed.

No writing assistance was utilized in the production of this manuscript.
} 
partitioning between intracellular and extracellular spaces. The primary determinants of renal excretion of potassium include sodium delivery to the distal nephron and urine flow, the renin-angiotensin-aldosterone system, vasopressin levels and acid-base status. Hormones and chemicals such as insulin, catecholamines and thyroid hormone affect intraversus extra-cellular partitioning of potassium, as well as acid-base status [15]. Dietary and serum potassium are not necessarily correlated given the tight control of serum potassium levels, and serum potassium does not necessarily reflect total body potassium stores, as subtle changes, even within the normal laboratory reference range, can occur with significant total body potassium depletion [16].

Adequate potassium intake, according to the US Panel on Dietary Reference Intake is $4.7 \mathrm{~g}$ $(120 \mathrm{mmol}) /$ day for adults, based on the assessment of the health benefits of potassium at this level on blood pressure, bone density and risk of kidney stones [16]. In general, dietary intake of potassium, at least in the USA and Canada, has been found to be much lower than this recommended value [16]. Further discussion regarding the different measurement tools for dietary potassium intake is given later, prior to discussion of the trials related to dietary potassium intake that use these tools.

\section{Experimentally induced hypokalemia \& glucose metabolism}

Experimental studies carried out in the 1960s-1980s aimed to determine why certain medical conditions (e.g., hyperaldosteronism and Bartter's syndrome) and medications (e.g., thiazide diuretics) associated with hypokalemia also seemed to be associated with glucose in tolerance. These studies determined that experimentally induced hypokalemia led to impaired glucose tolerance by reducing insulin secretion in response to glucose loads.

In order to test whether potassium was directly related to glucose metabolism, hyperglycemic clamp studies were performed in healthy volunteers in whom hypokalemia was induced; in several studies, hypokalemia was induced with thiazide diuretics $[17,18]$. In a study performed by Rowe et al., hypokalemia was induced with a low-potassium diet and subsequent use of sodium polystyrene sulfate [19]. In this study, the volunteers were determined to have normal glucose tolerance at baseline with a normal oral glucose tolerance test. Potassium depletion was documented with declines in plasma potassium levels ranging from $2.4-3.6 \mathrm{mEq} / \mathrm{dl}$, as well as declines in total body potassium measurements, as measured by a whole body counter. These declines in potassium levels were associated with declines in insulin release in response to hyperglycemia, which was experimentally maintained with glucose infusions. This study, consistent with other studies, found that the potassium depletion was associated with a decrease in pancreatic $\beta$-cell sensitivity to hyperglycemia with a reduction in insulin release.

Other studies were performed in which study participants who had hypokalemia induced through the use of thiazide diuretics and who demonstrated subsequent impaired glucose tolerance were subsequently given potassium supplements. After potassium supplementation was given, the defects in insulin release in response to glucose loads were corrected, thus further implicating the hypokalemia itself as being the causative agent of the glucose abnormality $[17,18]$.

\section{Antihypertensive drug effects on serum potassium \& diabetes risk}

\section{Thiazides}

Thiazide diuretics are commonly used for hypertension and have a common side effect of lowering serum potassium. These drugs have also been associated with increased risk of 
new-onset diabetes and, based on the results of several studies, it seems that these two effects are linked.

The association between thiazide diuretics and abnormalities in glucose metabolism came under closer scrutiny as recommendations were made for using thiazide diuretics as first-line agents for the treatment of hypertension [20].

Large-scale epidemiologic studies that studied the association of thiazide use and the development of diabetes showed conflicting results. A study combining the cohorts of Nurses' Health Study I and II and the Health Professionals Follow-up Study did find a significant increase in risk of diabetes with use of thiazide diuretics with an adjusted relative risk (RR) of 1.20 (95\% CI: 1.04-1.40) in older women, an RR of 1.51 (95\% CI: 1.15-1.98) in younger women and an RR of 1.31 (96\% CI: 1.07-1.60) in men [21]. However, data from other large cohorts did not find a statistically significant increased risk of incident diabetes with the use of thiazide diuretics [22-24].

Reviews of data from trials of antihypertensive agents have more consistently found associations between the use of thiazide diuretics and increased risk of diabetes. A network meta-analysis by Elliott et al. found that, compared with placebo, initial use of a diuretic was associated with an increased risk of diabetes, with an odds ratio (OR) of 1.30 (95\% CI: 1.07-1.58) (Table 1) [25]. Another quantitative review by Zillich et al. studied the association of thiazide use and glucose metabolism in 59 hyper tension trials that reported potassium and glucose measurements among participants placed on thiazides [10]. This review found a significant inverse association between potassium and glucose levels, with lower potassium levels corresponding to higher glucose levels. In the subset of studies that provided thiazide users with potassium supplements, this review found that there were smaller declines in potassium as well as smaller increases in glucose. A recent analysis of the Systolic Hypertension in Elderly Program clinical trial by Shafi et al. found that the change in serum potassium, which occurred after initiation of the thiazide diuretic chlorthalidone, was the primary mediator of the association between chlorthalidone use and risk of diabetes in this trial, with each $0.5 \mathrm{mEq}$ decrease in potassium being associated with a $45 \%$ higher adjusted risk of diabetes, primarily occurring within the first year of treatment [26].

Few trials have directly evaluated the effect of thiazides on glucose metabolism. A shortterm, 12-week trial by Eriksson et al. was performed in 26 nondiabetic, obese, hypertensive individuals comparing the effects of hydrochlorothiazide with the angiotensin II receptor blocker (ARB) candesartan and placebo on insulin resistance and visceral fat [27]. This crossover trial found that use of hydrochlorothiazide was associated with a reduction in insulin sensitivity and elevations in glycosylated hemoglobin, as well as increases in liver fat content. This study allowed for potassium supplementation, as needed, in those taking thiazides, but contrary to the aformentioned analyses of hypertension trial data, there was no correlation between potassium levels and the metabolic changes noted among the thiazide users [27]. In another trial by Bakris et al. of over 200 participants, two different antihypertensive combination regimens were compared for their effects on glucose metabolism. One treatment arm was placed on a combination of hydrochlorothiazide and the ARB losartan, while the other arm was placed on the angiotensin-converting enzyme inhibitor (ACE-I) trandolapril along with the calcium channel blocker verapamil SR [28]. This study, carried out over a period of 1 year, found that those on hydrochlorothiazide/ losartan had worsening of $2 \mathrm{~h}$ oral glucose tolerance tests compared with the baseline washout period, while those in the other treatment arm did not. There was also a significantly higher number of participants who developed diabetes by the end of the study among those on hydrochlorothiazide/losartan compared with trandoloapril/verapamil, with 
rates of 21 versus $7 \%$, respectively [28]. In addition, this study also did not find any correlation between changes in serum potassium and changes in glycemia. Further targeted large-scale trials are probably needed to determine if potassium and potassium supplementation could indeed be a causative factor and corrective factor, respectively, in the association between diuretic use and altered glucose metabolism.

\section{ACE-I/ARB}

The use of ACE-I and ARB have long had known benefits on the prevention of diabetesinduced nephropathy, but they are now also receiving greater attention for their possible role in the prevention of diabetes [29,30]. One of the many physiologic effects of the inhibitors of the renin-angiotensin-aldosterone system is the raising of serum potassium levels [31]. It is possible that their effects on glucose metabolism could, in part, be mediated through their effects on potassium.

Most of the evidence linking the use of ACE-I and ARBs with a decreased risk of diabetes has come from secondary analyses of hypertension trials. Post hoc analyses of data from the Antihypertensive and Lipid-Lowering Treatment to Prevent Heart Attack Trial (ALLHAT) trial found that participants taking lisinopril had a reduced incidence of new-onset diabetes compared with those on chlorthalidone (OR: 0.55 [95\%CI: 0.43-0.70]) [32]. Subsequent secondary or post hoc analyses of hypertension trials showed similar decreases in the risk of incident diabetes with ACE-I and ARB [33,34].

While the trial by Bakris et al. discussed previously did try to evaluate the effects of the ACE-I trandolapril on glycemia and diabetes risk, it was combined with another agent, verapamil, thus preventing an analysis of the direct effects of ACE inhibition on glycemia and diabetes risk. However, they did find that this combination did result in a much lower rate of incident diabetes at 1 year compared with hydrochlorothiazide/losartan [28]. The first randomized controlled trial designed to evaluate the effect of an ACE-I, ramipril, used alone on diabetes incidence showed no significant protective effect. The Diabetes Reduction Assessment with ramipril and rosiglitazone Medication (DREAM) trial enrolled participants with prediabetes (impaired fasting glucose or impaired glucose tolerance) and without cardiovascular disease, and found that ramipril did not lower the risk of incident diabetes over a median of 3 years but did increase the likelihood of regression to normoglycemia, a secondary outcome (Table 1) [29]. More recently, a similar trial, the Nateglinide And Valsartan in Impaired Glucose Tolerance Outcomes Research (NAVIGATOR) trial, was carried out using the ARB valsartan. This study combined the use of valsartan along with lifestyle modification in participants with known impaired glucose tolerance and known cardiovascular disease or risk factors for cardiovascular disease. This study did find a protective effect on diabetes risk among users of valsartan compared with placebo over a median follow-up period of 5 years (hazard ratio [HR]: 0.86 [95\% CI: 0.80-0.92]) (Table 1) [30].

Recent meta-analyses evaluating the association of the use of ACE-I/ARBs and the risk of incident diabetes have included data from trials in which diabetes was either a primary or secondary outcome. These meta-analyses have also found a statistically significantly reduced risk of diabetes among users of ACE-I/ARBs, with ORs ranging from 0.7 to 0.8 compared with placebo or active controls [33,34].

As mentioned previously, whether or not there is a direct association between these medications' effects on potassium and glucose has not been systematically evaluated. There are several potential ways in which ACE-I and ARB could affect glucose metabolism, including their direct vascular effects on organs that metabolize glucose and their antioxidant properties, as well as their effect on the production of other hormones - 
including bradykinin, angiotensin II, aldosterone and nitric oxide, all of which have effects on insulin function $[35,36]$. One study by Santoro et al. studied the direct effect of the ACEI cilazapril on glucose tolerance, potassium level and insulin effects, utilizing $2 \mathrm{~h}$ oral glucose tolerance tests and euglycemic insulin clamp tests in 20 participants with hypertension and normal glucose tolerance [37]. This study found that therapy with lowdose cilazapril over 3 months was associated with a 'blunted fall in potassium' in response to insulin, as well as improved glucose tolerance in response to a glucose challenge. Further studies are needed to determine if the effects of ACE-I/ARB on potassium are directly associated with their beneficial effects on diabetes risk.

\section{Serum potassium \& diabetes risk independent of antihypertensive drug effects}

Two observational studies have examined the association between serum potassium and risk of diabetes independent of antihypertensive use. The first study by Chatterjee et al. analyzed data from the Atherosclerosis Risk in Communities (ARIC) Study, a prospective cohort of middle-aged adults. This study analyzed data from participants who did not have diabetes at baseline. In separate multivariable analyses, they either adjusted for use of antihypertensive agents or excluded participants on antihypertensive agents. In both analyses, investigators found a statistically significant inverse association between serum potassium and risk of incident diabetes, with lower potassium levels predicting increased risk. Those participants with a serum potassium in the lowest quartile $(<4.0 \mathrm{mEq} / \mathrm{l})$ had an adjusted HR of incident diabetes of 1.64 (95\% CI: 1.29-2.08) over 9 years of follow-up, independent of traditional risk factors for diabetes, including the presence of hypertension and use of antihypertensive agents [13]. In further analyses, they found that this increased risk of diabetes associated with lower potassium levels, while present in both African-Americans and whites, may be stronger in African-Americans [38].

The second study by Heianza et al. examined the association between serum potassium, independent of antihypertensive use, and diabetes risk in a Japanese cohort [14]. This study included Japanese men who were not on antihypertensive agents and who did not have diabetes at baseline. They found very similar results to the ARIC study, with those in the lowest tertile of serum potassium (serum potassium $2.8-3.9 \mathrm{mEq} / \mathrm{l}$ ) having an adjusted HR of incident diabetes of 1.57 (95\% CI: 1.15-2.15) [14]. Similar to the analyses of data from the Systolic Hypertension in the Elderly Program (SHEP) trial, this study found that for each $0.5 \mathrm{mEq} / \mathrm{l}$ decrease in serum potassium, there was a $45 \%$ increased risk of diabetes.

Further studies are needed to determine whether this association between low-to-normal serum potassium and increased diabetes risk is direct and causal or if this association is mediated by other factors that regulate serum potassium.

\section{Dietary potassium \& diabetes risk}

The association between dietary potassium intake and glucose metabolism has been studied in different epidemiologic cohorts using different methods of measurement and with varied results. Dietary potassium has been measured in different ways. Measurements based on dietary history are the most commonly used in epidemiologic studies. Food frequency questionnaires, $24 \mathrm{~h}$ dietary recalls and food diaries are examples of a few of them. One study measured and compared the accuracy of nutrient intake estimations based on these different tools compared with weighed food records as well as $24 \mathrm{~h}$ urine measurements. In this study, estimations of potassium intake based on food frequency questionnaires were the most accurate compared with other questionnaires after adjustment for total energy intake [39]. However, the reliability and validity of the different tools based on dietary history can 
vary by population [40]. $24 \mathrm{~h}$ urinary potassium levels have been used as a fairly accurate measure of dietary potassium intake, with a Pearson correlation coefficient of approximately 0.8 compared with measured nutrient intake, and have also been used in epidemiologic studies $[16,41]$. However, these measurements are more costly and time-consuming for participants [41,42]. These measurements also can vary by population [43]. Most accurate, but also most costly, are feeding studies that utilize meals specially prepared in metabolic kitchens with calculated amounts of nutrients in them [16,44].

Some studies have used the aforementioned measurements of potassium intake to evaluate the association of dietary potassium and diabetes risk. Other studies have evaluated the associations of fruit and vegetable intake, the primary sources of dietary potassium, on diabetes risk without separating out the individual effects of the different vitamins, minerals and electrolytes contained in the fruits and vegetables, on these associations. No feeding studies that we are aware of have yet been performed to assess the direct association between dietary potassium and diabetes risk.

\section{Overall potassium intake \& diabetes risk}

Studies examining the association between dietary potassium intake and diabetes risk vary in the tools that they use to measure dietary potassium intake. The results of these studies have been mixed but have had mostly null findings, possibly due to the tools used for measurement or due to varied effects in different populations. In addition, in the few studies that have examined the association between dietary potassium intake and diabetes risk, dietary potassium intake was on average lower than the adequate intake level $[13,45]$. One study by Hu et al., which was carried out in a Finnish, primarily white, population, examined the association between urinary potassium and diabetes risk and found no significant association, with those in the highest quintile of potassium intake having measures of urinary potassium excretion of $94 \mathrm{mmol} / 24 \mathrm{~h} \mathrm{[45].} \mathrm{A} \mathrm{study} \mathrm{based} \mathrm{on} \mathrm{the} \mathrm{ARIC}$ cohort found no significant association between dietary potassium, as measured by a food frequency questionnaire, and diabetes risk, with average dietary potassium intake levels of less than $2700 \mathrm{mg} /$ day [13]. A study by Colditz et al. analyzed data from the Nurses' Health Study, a primarily white female population, and found a significant association between dietary potassium intake and diabetes risk, adjusting for other traditional risk factors, but this association was diminished when other dietary measures were included in their models. Furthermore, the absolute amount of dietary potassium consumed in this cohort was not described [46]. Further studies using standardized, more reliable and more accurate measurements of potassium intake are needed to be able to compare results from different studies and to better assess the association between dietary potassium intake and diabetes risk. Studies are also needed in which participants have adequate intake of dietary potassium, in order to evaluate the effect of this level of potassium intake on diabetes outcomes.

\section{Fruit \& vegetable intake \& diabetes risk}

Several studies have been carried out evaluating the association between the intake of certain fruits and vegetables on diabetes risk. One epidemiologic study by Liese et al. found that increased adherence to a Dietary Approaches to Stop Hypertension (DASH) diet, a diet that is rich in fruits and vegetables and high in potassium, based on a 1-year food frequency questionnaire, was associated with lowered risk of diabetes in whites but not AfricanAmericans [47]. Those whites in the highest tertile of adherence to a DASH diet had an adjusted OR of incident diabetes of 0.31 (95\% CI: 0.13-0.75) compared with those in the lowest tertile. However, a recent trial, carried out over 4 months, found that the DASH diet alone did not affect insulin sensitivity or glucose measures over the trial period, but that the DASH diet combined with caloric restriction and exercise did result in improvement in both 
insulin sensitivity and glucose levels [48]. A recent meta-analysis and review by Carter $\mathrm{et} \mathrm{al}$. studied the association between the intake of fruits and vegetables and risk of incident diabetes in prospective cohort studies. This meta-analysis included six studies and found a significant association between greater intake (1.35 servings or more per day) of green, leafy vegetables, which are foods that are potassium rich, and lower risk of diabetes, with an adjusted HR of 0.86 (95\% CI: 0.77-0.97) compared with those with lower intake $(0.2$ servings or less per day) [49]. However, this study was not able to examine the specific micronutrients that contributed to this association. This review also concluded that there were very few studies that adequately evaluated the association between intake of particular fruits and vegetables and diabetes risk and concluded that further studies are needed to support specific dietary recommendations for diabetes prevention. Finally, diets that are high in vegetable content are also likely to have a low glycemic index, which should be taken into account when assessing their association with diabetes risk. Further trials are needed to clarify whether or not dietary modifications with foods high in potassium or high in fruits and vegetables, without changes in weight, can affect diabetes risk in the long term.

\section{Genetic studies of potassium channels \& diabetes risk}

The potential for potassium to play a role in diabetes risk and as a potential intervention for prevention or treatment is enhanced by the finding that mutations in genes coding for potassium channels affecting insulin secretion have been discovered. In particular the KCNJ11 gene contains code for the potassium channel on pancreatic $\beta$-cells, which are involved with insulin secretion. A certain mutation of this gene has been found to lead to neonatal diabetes, but a less drastic single nucleotide polymorphism has been found to be associated with Type 2 diabetes, with an OR of approximately 1.14 [6]. Another gene, $K C N Q 1$, also encodes for a potassium channel involved in insulin secretion. Mutations of this gene have been associated with increased risk of Type 2 diabetes in primarily Asian populations [6,50,51]. The influence of serum and/or dietary potassium on the function of these channels is not clear, but further studies should be undertaken to determine if these levels influence the function of these channels in their normal state and in their genetically mutated states.

\section{Expert commentary \& five-year view}

Most of the literature reviewed here has been secondary analyses of clinical trial data or observational data that have found associations between drugs that affect potassium levels, serum potassium, dietary potassium and foods that are high in potassium with diabetes risk. Table 1 summarizes the associations found between markers of potassium and risk of incident diabetes as well as the level of evidence supporting these associations. Over 20 years ago, experimental hyperglycemic clamp studies demonstrated that potassium supplementation did improve insulin sensitivity of participants who had experimentally induced hypokalemia, but no randomized controlled trial has tested this effect in a larger population. The studies evaluating the effects of ACE inhibitors and ARBs on diabetes risk did not isolate the potential effect of changes in potassium levels on these associations. Furthermore, no clinical trials to our knowledge have evaluated the impact on potassium supplementation, either dietary or pharmacologic, in any population, on glucose metabolism.

Potassium is a vital electrolyte. Both high and low levels are already associated with various medical conditions, including hypertension, cardiac arrhythmias, osteoporosis and nephrolithiasis [16]. It is plausible, given the evidence presented in this article, that potassium also has a significant role in diabetes mellitus. In observational studies, there are fairly strong associations between serum potassium and diabetes risk, with lower serum levels associated with increased risk. In observational studies, there is a suggestion that 
dietary potassium may be associated with diabetes risk. In studies involving both serum and dietary potassium, there is a suggestion that their effects on diabetes risk could vary based on race. These racial differences suggest that there could be genetic factors that could affect the association between lower potassium levels and diabetes risk. This could also mean that genetic factors may potentially influence the response to interventions designed to raise potassium levels.

Since most studies examining the association between potassium and diabetes risk have been observational in nature, experimental studies are needed to help clarify the effect of both serum and dietary potassium on diabetes risk. Interventions with potassium supplementation, either dietary or pharmacologic, should be systematically evaluated as potential novel intervention for the prevention and treatment of diabetes, particularly in high-risk populations.

\section{Acknowledgments}

Hsin-Chieh Yeh and Frederick Brancati were supported by the NIDDK Diabetes Research and Training Center (grant P60 DK079637). Frederick Brancati was supported by a grant from the NIH/NIDDK (grant K24 DK62222).

\section{References}

Papers of special note have been highlighted as:

- of interest

1. Dall T, Mann SE, Zhang Y, et al. Economic costs of diabetes in the U.S in 2007. Diabetes Care. 2008; 31(3):596-615. [PubMed: 18308683]

2. Wild S, Roglic G, Green A, Sicree R, King H. Global prevalence of diabetes: estimates for the year 2000 and projections for 2030. Diabetes Care. 2004; 27(5):1047-1053. [PubMed: 15111519]

3. Stern MP, Haffner SM. Body fat distribution and hyperinsulinemia as risk factors for diabetes and cardiovascular disease. Arteriosclerosis. 1986; 6(2):123-130. [PubMed: 3513749]

4. Martini LA, Catania AS, Ferreira SR. Role of vitamins and minerals in prevention and management of Type 2 diabetes mellitus. Nutr Rev. 2010; 68(6):341-354. [PubMed: 20536779]

5. Hectors TL, Vanparys C, van der Ven K, et al. Environmental pollutants and Type 2 diabetes: a review of mechanisms that can disrupt $\beta$ cell function. Diabetologia. 2011; 54(6):1273-1290. [PubMed: 21442161]

6. Grant, RW.; Moore, AF.; Florez, JC. Diabetes Care. Vol. 32. 2009. Genetic architecture of Type 2 diabetes: recent progress and clinical implications; p. 1107-1114. Review of genetic mutations associated with increased diabetes risk.

7. Corry DB, Tuck ML. The effect of aldosterone on glucose metabolism. Curr Hypertens Rep. 2003; 5(2):106-109. [PubMed: 12642008]

8. Conn JW. Hypertension, the potassium ion and impaired carbohydrate tolerance. N Engl J Med. 1965; 273(21):1135-1143. [PubMed: 5841677]

9. Carter BL, Einhorn PT, Brands M, et al. Thiazide-induced dysglycemia: call for research from a working group from the National Heart, Lung, and Blood institute. Hypertension. 2008; 52:30-36. [PubMed: 18504319]

10. Zillich AJ, Garg J, Basu S, Bakris GL, Carter BL. Thiazide diuretics, potassium, and the development of diabetes: a quantitative review. Hypertension. 2006; 48(2):219-224. • Quantitative review of thiazide-induced hypokalemia and effects on glucose. [PubMed: 16801488]

11. Rapoport MI, Hurd HF. Thiazide-induced glucose intolerance treated with potassium. Arch Intern Med. 1964; 113:405-408. [PubMed: 14096393]

12. Jackson JH, Sobolski J, Krienke R, Wong KS, Frech-Tamas F, Nightengale B. Blood pressure control and pharmacotherapy patterns in the United States before and after the release of the Joint 
National Committee on the Prevention, Detection, Evaluation, and Treatment of High Blood Pressure (JNC 7) guidelines. J Am Board Fam Med. 2008; 21(6):512-521. [PubMed: 18988718]

13. Chatterjee R, Yeh HC, Shafi T, et al. Serum and dietary potassium and risk of incident Type 2 diabetes mellitus: the Atherosclerosis Risk in Communities (ARIC) study. Arch Intern Med. 2010; 170:1745-1751. [PubMed: 20975023]

14. Heianza Y, Hara S, Arase Y, et al. Low serum potassium levels and risk of Type 2 diabetes: the Toranomon Hospital Health Management Center Study 1 (TOPICS1). Diabetologia. 2011; 54(4): 762-766. [PubMed: 21212932]

15. Evans KJ, Greenberg A. Hyperkalemia: a review. J Intensive Care Med. 2005; 20(5):272-290. [PubMed: 16145218]

16. In: Dietary Reference Intakes for Water, Potassium, Sodium, Chloride, and Sulfate. The National Academies Press; Washington DC, USA: 2004. Panel on Dietary Reference Intakes for Electrolytes and Water; The Standing Committee on the Scientific Evaluation of Dietary Reference Intakes; National Academy of Sciences. Chapter 5: Potassium; p. 186-268. Informative chapter on potassium, potassium handling and health effects of potassium.

17. Gorden P. Glucose intolerance with hypokalemia Failure of short-term potassium depletion in normal subjects to reproduce the glucose and insulin abnormalities of clinical hypokalemia. Diabetes. 1973; 22(7):544-551. [PubMed: 4719191]

18. Helderman JH, Elahi D, Andersen DK. Prevention of the glucose intolerance of thiazide diuretics by maintenance of body potassium. Diabetes. 1983; 32(2):106-111. - Trial with hyperglycemia clamps evaluating the effects of hypokalemia, induced with thiazides, on glucose metabolism and effects on subsequent potassium supplementation. [PubMed: 6337892]

19. Rowe JW, Tobin JD, Rosa RM, Andres R. Effect of experimental potassium deficiency on glucose and insulin metabolism. Metabolism. 1980; 29(6):498-502. - Trial with hyperglycemic clamps evaluating the effects of hypokalemia not induced with thiazide diuretics on serum and total body potassium and glucose metabolism. [PubMed: 6991855]

20. The ALLHAT Officers and Coordinators for the ALLHAT Collaborative Research Group. Major outcomes in high-risk hypertensive patients randomized to angiotensin-converting enzyme inhibitor or calcium channel blocker vs diuretic: the Antihypertensive and Lipid-Lowering Treatment to Prevent Heart Attack Trial (ALLHAT). JAMA. 2002; 288(23):2981-2997. [PubMed: 12479763]

21. Taylor EN, Hu FB, Curhan GC. Antihypertensive medications and the risk of incident Type 2 diabetes. Diabetes Care. 2006; 29(5):1065-1070. [PubMed: 16644638]

22. Burke TA, Sturkenboom MC, Ohman-Strickland PA, Wentworth CE, Rhoads GG. The effect of antihypertensive drugs and drug combinations on the incidence of new-onset Type 2 diabetes mellitus. Pharmacoepidemiol Drug Saf. 2007; 16(9):979-987. [PubMed: 17605137]

23. Gress TW, Nieto FJ, Shahar E, Wofford MR, Brancati FL. Hypertension and antihypertensive therapy as risk factors for Type 2 diabetes mellitus Atherosclerosis Risk in Communities Study. N Engl J Med. 2000; 342(13):905-912. [PubMed: 10738048]

24. Donahue RP, Abbott RD, Wilson PW. Effect of diuretic use on the development of diabetes mellitus. The Framingham study. Horm Metab Res Suppl. 1990; 22:46-48. [PubMed: 2391060]

25. Elliott WJ, Meyer PM. Incident diabetes in clinical trials of antihypertensive drugs: a network meta-analysis. Lancet. 2007; 369(9557):201-207. • Network meta-analysis evaluating the effects of antihypertensive agents on diabetes risk. [PubMed: 17240286]

26. Shafi T, Appel LJ, Miller ER, Klag MJ, Parekh RS. Changes in serum potassium mediate thiazideinduced diabetes. Hypertension. 2008; 52:1022-1029. [PubMed: 18981326]

27. Eriksson JW, Jansson PA, Carlberg B, et al. Hydrochlorothiazide, but not Candesartan, aggravates insulin resistance and causes visceral and hepatic fat accumulation: the Mechanisms for the Diabetes Preventing Effect of Candesartan (MEDICA) study. Hypertension. 2008; 52(6):10301037. [PubMed: 18981327]

28. Bakris G, Molitch M, Hewkin A, et al. Differences in glucose tolerance between fixed-dose antihypertensive drug combinations in people with metabolic syndrome. Diabetes Care. 2006; 29(12):2592-2597. [PubMed: 17130190] 
29. Bosch J, Yusuf S, Gerstein. Effect of ramipril on the incidence of diabetes. N Engl J Med. 2006; 355(15):1551-1562. • Randomized controlled trial assessing the effect of the angiotensinconverting enzyme inhibitor ramipril on diabetes risk. [PubMed: 16980380]

30. McMurray JJ, Holman RR, Haffner SM. Effect of valsartan on the incidence of diabetes and cardiovascular events. N Engl J Med. 2010; 362(16):1477-1479. • Randomized controlled trial assessing the effect of the angiotensin receptor blocker valsartan on diabetes risk. [PubMed: 20228403]

31. Weir MR, Rolfe M. Potassium homeostasis and renin-angiotensin-aldosterone system inhibitors. Clin J Am Soc Nephrol. 2010; 5(3):531-548. [PubMed: 20150448]

32. Barzilay JI, Davis BR, Cutler JA, et al. Fasting glucose levels and incident diabetes mellitus in older nondiabetic adults randomized to receive 3 different classes of antihypertensive treatment: a report from the Antihypertensive and Lipid-Lowering Treatment to Prevent Heart Attack Trial (ALLHAT). Arch Intern Med. 2006; 166(20):2191-2201. [PubMed: 17101936]

33. Andraws R, Brown DL. Effect of inhibition of the renin-angiotensin system on development of Type 2 diabetes mellitus (meta-analysis of randomized trials). Am J Cardiol. 2007; 99(7):10061012. [PubMed: 17398202]

34. Tocci G, Paneni F, Palano F, et al. Angiotensin-converting enzyme inhibitors, angiotensin II receptor blockers and diabetes: a meta-analysis of placebo-controlled clinical trials. Am J Hypertens. 2011; 24(5):582-590. [PubMed: 21331058]

35. McGuire DK, Winterfeld JR, Rytlewski JA, et al. Blocking the renin-angiotensin- aldosterone system to prevent diabetes mellitus. Vasc Dis Res. 2008; 5(1):59-66.

36. Hershon KS. Mechanistic and clinical aspects of renin-angiotensin-aldosterone system (RAAS) blockade in the prevention of diabetes and cardiovascular disease. Endocr Pract. 2011; 17(3):430 440. [PubMed: 21454245]

37. Santoro D, Natali A, Palombo C, et al. Effects of chronic angiotensin converting enzyme inhibition on glucose tolerance and insulin sensitivity in essential hypertension. Hypertension. 1992; 20(2): 181-191. [PubMed: 1639459]

38. Chatterjee R, Yeh HC, Shafi T, et al. Serum potassium and the racial disparity in diabetes risk: the Atherosclerosis Risk in Communities (ARIC) Study. Am J Clin Nutr. 2011; 93(5):1087-1091. [PubMed: 21367942]

39. Bingham SA, Day NE. Using biochemical markers to assess the validity of prospective dietary assessment methods and the effect of energy adjustment. Am J Clin Nutr. 1997; 65(4 Suppl): 1130S-1137S. [PubMed: 9094909]

40. Liu K, Slattery M, Jacobs D Jr. A study of the reliability and comparative validity of the CARDIA dietary history. Ethn Dis. 1994; 4(1):15-27. [PubMed: 7742729]

41. Tasevska N, Runswick SA, Bingham SA. Urinary potassium is as reliable as urinary nitrogen for use as a recovery biomarker in dietary studies of free living individuals. J Nutr. 2006; 136(5): 1334-1340. [PubMed: 16614426]

42. Bingham SA, Gill C, Welch A, et al. Validation of dietary assessment methods in the UK arm of EPIC using weighed records, and 24-hour urinary nitrogen and potassium and serum vitamin C and carotenoids as biomarkers. Int J Epidemiol. 1997; 26(Suppl. 1):S137-S151. [PubMed: 9126542]

43. Turban S, Miller ER, Ange B, Appel LJ. Racial differences in urinary potassium excretion. J Am Soc Nephrol. 2008; 19(7):1396-1402. [PubMed: 18579642]

44. Conlin PR, Chow D, Miller ER, et al. The effect of dietary patterns on blood pressure control in hypertensive patients: results from the Dietary Approaches to Stop Hypertension (DASH) trial. Am J Hypertens. 2000; 13(9):949-955. [PubMed: 10981543]

45. Hu G, Jousilahti P, Peltonen M, Lindström J, Tuomilehto J. Urinary sodium and potassium excretion and the risk of Type 2 diabetes: a prospective study in Finland. Diabetologia. 2005; 48(8):1477-1483. [PubMed: 15971060]

46. Colditz GA, Manson JE, Stampfer MJ, Rosner B, Willett WC, Speizer FE. Diet and risk of clinical diabetes in women. Am J Clin Nutr. 1992; 55(5):1018-1023. [PubMed: 1315120] 
47. Liese AD, Nichols M, Sun X, et al. Adherence to the DASH diet is inversely associated with incidence of Type 2 diabetes: the insulin resistance atherosclerosis study. Diabetes Care. 2009; 32(8):1434-1436. [PubMed: 19487638]

48. Blumenthal JA, Babyak MA, Sherwood A, et al. Effects of the dietary approaches to stop hypertension diet alone and in combination with exercise and caloric restriction on insulin sensitivity and lipids. Hypertension. 2010; 55(5):1199-1205. [PubMed: 20212264]

49. Carter, P.; Gray, LJ.; Troughton, J.; Khunti, K.; Davies, MJ. BMJ. Vol. 341. 2010. Fruit and vegetable intake and incidence of Type 2 diabetes mellitus: systematic review and meta-analysis; p. c4229• Meta-analysis of the effects of fruits and vegetables in diet on diabetes risk.

50. Been LF, Ralhan S, Wander GS, et al. Variants in KCNQ1 increase Type II diabetes susceptibility in South Asians: a study of 3,310 subjects from India and the US. Med Genet. 2011; 12:18.

51. Zhou JB, Yang JK, Zhao L, Xin Z. Variants in KCNQ1, AP3S1, MAN2A1, and ALDH7A1 and the risk of Type 2 diabetes in the Chinese Northern Han population: a case-control study and metaanalysis. Med Sci Monit. 2010; 16(6):BR179-BR183. [PubMed: 20512086]

\section{Website}

101. International Diabetes Federation. Position statement - diabetes and obesity. www.idf.org/position-statement-diabetes-and-obesity 
Key issues

- Experimentally induced hypokalemia has been associated with reduced insulin sensitivity and the development of impaired glucose tolerance in hyperglycemic clamp studies.

- Use of thiazide diuretics is associated with increased risk of diabetes, and this association is most likely mediated by the hypokalemic effects of the thiazide diuretics.

- Use of angiotensin II receptor blockers (ARBs) is associated with reduced risk of diabetes.

- ARBs cause increases in serum potassium levels. Whether or not there is an association between the potassium-sparing effects of the ARBs and reduced diabetes risk has not been evaluated.

- Low normal serum potassium, independent of the use of antihypertensive agents, is associated with an increased risk of incident diabetes in prospective cohort studies.

- Low dietary potassium intake has not been clearly associated with increased risk of incident diabetes.

- Higher intake of potassium-rich foods has been associated with a reduced risk of diabetes.

- Randomized controlled trials are needed to determine if potassium supplementation, either dietary or pharmacologic, will impact short-term measures of glycemia and long-term risk of diabetes. 


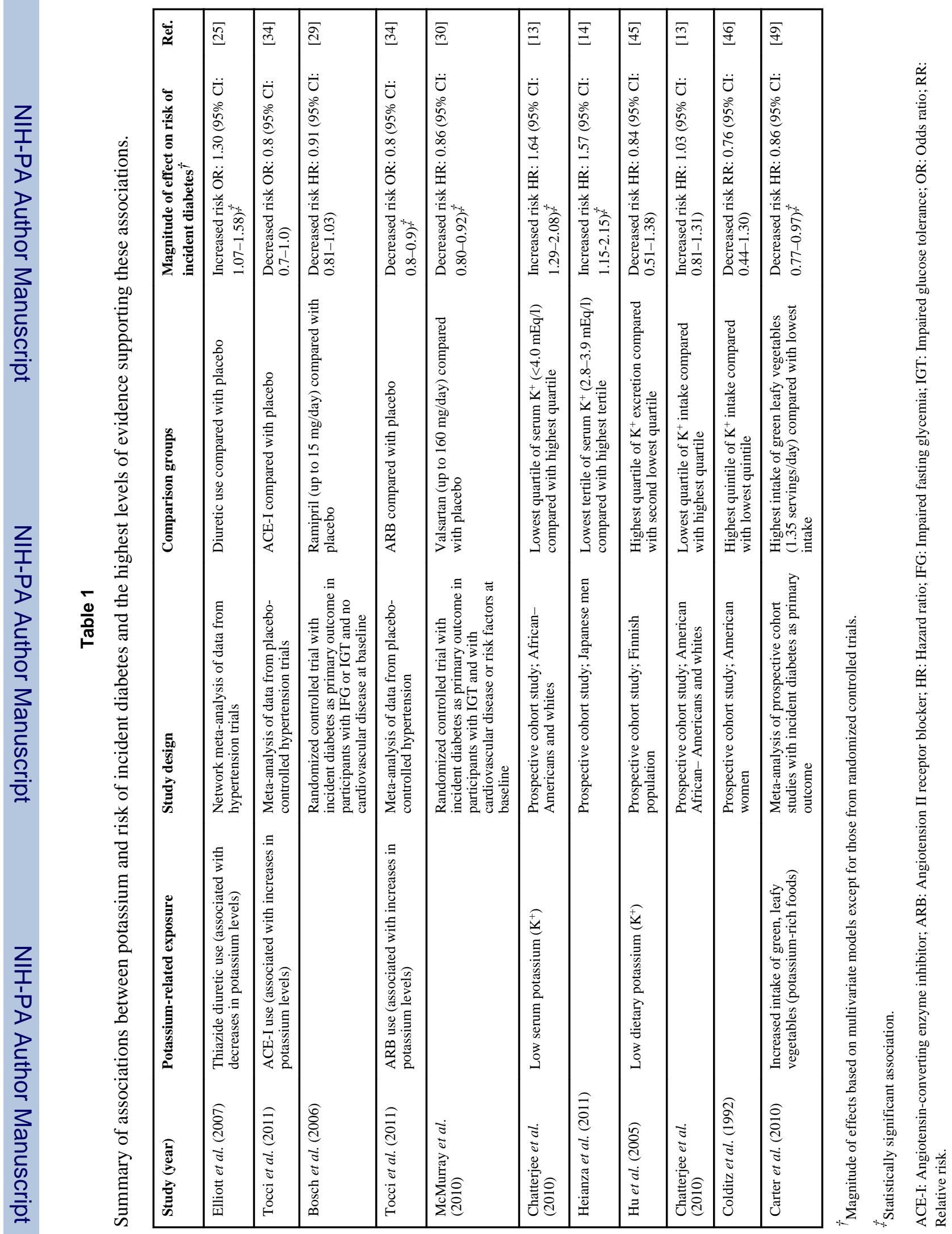

Expert Rev Endocrinol Metab. Author manuscript; available in PMC 2012 July 1. 